\title{
INEQUALITIES FOR EIGENVALUES OF SELFADJOINT OPERATORS
}

\author{
STEPHEN M. HOOK
}

Dedicated to the loving memory of my father

\begin{abstract}
We establish several inequalities for eigenvalues of selfadjoint operators in Hilbert space. The results are quite general. In particular, let $\Omega$ be a region in $\mathbf{R}^{n}, \partial \Omega$ its boundary and $\Delta$ the Laplace operator in $\mathbf{R}^{n}$. Let $p(x)$ be a polynomial of degree $m$ having nonnegative real coefficients. We show that if the problems

(1) $-\Delta u=\lambda u$ in $\Omega ; u=0$ on $\partial \Omega$;

(2) $p(-\Delta) v=\mu v$ in $\Omega ; v$ and its first $m-1$ derivatives $=0$ on $\partial \Omega$; and

(3) $(-\Delta)^{m} w=\nu w$ in $\Omega ; w$ and its first $m-1$ derivatives $=0$ on $\partial \Omega$ are selfadjoint with discrete spectra of finite multiplicity $\lambda_{1} \leq \lambda_{2} \leq \cdots$ etc. then

(4) $p\left(\Gamma_{i}^{1 / m}\right) \geq \mu_{i} \geq p\left(\lambda_{i}\right)$ for each index $i$. The set of problems (1), (2), (3) and the result (4) is only one example of our more general result.

The above problems (1), (2), and (3) can be thought of as related through the single operator given by the Laplacian. We also establish results for eigenvalues for unrelated operators. Let $A, B$, and $A+B$ be selfadjoint on domains $D_{A}$, $D_{B}$, and $D_{A+B}$ with $D_{A+B} \subseteq D_{A} \cap D_{B}$. If $A, B$, and $A+B$ have discrete spectra $\left\{\lambda_{i}\right\}_{i=1}^{\infty},\left\{\mu_{i}\right\}_{i=1}^{\infty}$ and $\left\{\Gamma_{i}\right\}_{i=1}^{\infty}$ arranged in ascending order, as above, then inequality
\end{abstract}

(5) $\sum_{i=1}^{n} \Gamma_{i} \geq \sum_{i=1}^{n}\left(\lambda_{i}+\nu_{i}\right)$ is established for each positive integer $n$.

\section{INTRODUCTION}

Let $H$ be any Hilbert space. Suppose that $A$ is a linear operator in $H$. Consider the three dense subspaces of $H, D_{3} \subseteq D_{2} \subseteq D_{1} \subseteq H$. Let $p$ be any polynomial of degree $m$ in one variable with real nonnegative coefficients. Suppose that the operators

$$
\begin{gathered}
B_{1}=A \text { restricted to } D_{1}, \\
B_{2}=p(A) \text { restricted to } D_{2},
\end{gathered}
$$

and

$$
B_{3}=A^{m} \text { restricted to } D_{3}
$$

are selfadjoint with discrete nonnegative spectra. (Here $p(A)$ is the operator obtained by formally substituting the operator $A$ for the indeterminate.)

Received by the editors December 1, 1986 and, in revised form, November 6, 1987 and June 6, 1988.

1980 Mathematics Subject Classification (1985 Revision). Primary 35P15. (C) 1990 American Mathematical Society
$0002-9947 / 90 \$ 1.00+\$ .25$ per page 
Denote the spectrum of $B_{1}$ by $\lambda_{1} \leq \lambda_{2} \leq \cdots$, the spectrum of $B_{2}$ by $\mu_{1} \leq \mu_{2} \leq \cdots$ and the spectrum of $B_{3}$ by $\nu_{1} \leq \nu_{2} \leq \nu_{3} \leq \cdots$.

In the event that $B_{2}=p\left(B_{1}\right)$, the spectral mapping theorem implies that $\mu_{i}=p\left(\lambda_{i}\right)$ for each $i$. Likewise if $B_{3}=B_{1}^{m}$ then we have $\lambda_{i}^{m}=\nu_{i}$ for all $i$. For unbounded operators there are generally choices of $D_{1}, D_{2}$, and $D_{3}$ so that $B_{2} \neq p\left(B_{1}\right)$ or $B_{3} \neq B_{1}^{m}$. When this happens there is no general relationship between the eigenvalues of $B_{1}, B_{2}$, and $B_{3}$.

In this paper we establish conditions on the domains $D_{1}, D_{2}$, and $D_{3}$ and the operator $A$ for which the inequality $p\left(\lambda_{i}\right) \leq \mu_{i} \leq p\left(\left(\nu_{i}\right)^{1 / m}\right)$ holds. As an example, we establish that the eigenvalues for the three problems

$$
\begin{gathered}
-\Delta u=\lambda u \quad \text { in } \Omega ; \quad u=0 \quad \text { on } \partial \Omega, \\
p(-\Delta) v=\mu v \quad \text { in } \Omega ; \quad v=\frac{\partial v}{\partial n}=\cdots=\frac{\partial^{m-1} v}{\partial n^{m-1}}=0 \quad \text { on } \partial \Omega,
\end{gathered}
$$

and

$$
(-\Delta)^{m} w=\nu w \quad \text { in } \Omega ; \quad w=\frac{\partial w}{\partial n}=\cdots=\frac{\partial^{m-1} w}{\partial n^{m-1}}=0 \quad \text { in } \partial \Omega
$$

satisfy the inequality

$$
p\left(\lambda_{i}\right)<\mu_{i} \leq p\left(\left(\nu_{i}\right)^{1 / m}\right) .
$$

Here $p$ is any polynomial with nonnegative real coefficients of degree $m$, $\Omega$ is any open region in $\mathbf{R}^{n}$ for which problems (1.4), (1.5), and (1.6) are selfadjoint with discrete spectra, $\partial \Omega$ is the boundary set of $\Omega, \Delta$ is the Laplace operator and $\partial / \partial n$ is the normal outward directed derivative on $\partial \Omega$.

Results of this type have been established before by Payne [7], Diaz [4], and Weinstein [8] (as well as others) but not with the generality discussed here.

We also establish for problems

$$
\begin{array}{ll}
A u=\lambda u & \text { for } u \in D_{A}, \\
B v=\mu v & \text { for } v \in D_{B}
\end{array}
$$

and

$$
(A+B) w=\Gamma w \text { for } w \in D_{A+B},
$$

where $A, B$, and $A+B$, are selfadjoint operators in a Hilbert space $H$, that $\sum_{i=1}^{n} \Gamma_{i} \geq \sum_{i=1}^{n}\left(\lambda_{i}+\mu_{i}\right)$ for each positive integer $n$. This result is a generalization of an earlier result of Payne [7] and is applicable for choices of $A$ and $B$ which are not related through the operator $A$ in (1.1)-(1.3).

\section{AN INEQUALITY RELATING POWERS OF AN OPERATOR}

Ultimately we will use the so-called "mini-max principle". This will require estimates for quantities of the form: $\left(A^{k} u, u\right)$ in terms of $\left(A^{m} u, u\right)$ where 
$0 \leq k \leq m$ are integers, $u$ is a vector in the domain of $A^{m}$ and $(\cdot, \cdot)$ is the inner product. The proof is based on some work of Chen [2] but its results have been improved slightly as well as generalized. In this paper the quantity $0^{0}=1$.

Theorem 1. Let $V$ be a real or complex inner product space with inner product $(\cdot, \cdot)$. Let $D$ be a linear submanifold of $V$ and let $A: D \rightarrow V$ be a linear operator in $V$. Let $n$ be a positive integer and $u$ be a fixed vector in the domain of $A^{n}$. Suppose that for all integers $r$ and $p$ which satisfy $0 \leq r \leq p \leq n$, the equality $\left|\left(A^{p} u, u\right)\right|=\left|\left(A^{p-r} u, A^{r} u\right)\right|$ holds. Then

(i) for the integer $k$ and each even integer $m$ which satisfy $0 \leq k \leq m \leq n$, the inequality

$$
\left|\left(A^{k} u, u\right)\right| \leq\left|\left(A^{m} u, u\right)\right|^{k / m}(u, u)^{(m-k) / m}
$$

holds.

(ii) Inequality (2.1) is satisfied for each integer $k$ and odd integer $m$ which satisfy $0 \leq k \leq m \leq n$ if in addition to the above hypotheses there is a finite collection of operators $\left\{B_{j}: j=1,2, \ldots, M\right\}$ in $V$ which satisfy the following: For each pair of integers $r$ and $p$ so that $1 \leq r \leq p \leq n$ the equality

$$
\left|\left(A^{p} u, u\right)\right|=\left|\sum_{j=1}^{M}\left(B_{j} A^{p-r} u, B_{j} A^{r-1} u\right)\right|
$$

holds.

Remarks. (i) If $A$ is any (not necessarily bounded) selfadjoint operator then part (i) of Theorem 1 is satisfied and thus inequality (2.1) holds for all even integers $m$.

(ii) If $A$ is any (not necessarily bounded) nonnegative selfadjoint operator then part (ii) of Theorem 1 is satisfied by taking $M=1$ and letting $B_{1}$ be any square root of $A$. Hence, for nonnegative selfadjoint operators inequality (2.1) holds for all integers $m$.

(iii) Inequality (2.1) is an equality if $A$ is selfadjoint and $u$ is any eigenvector of $A$.

(iv) As we shall see shortly, the proof of Theorem 1 depends on the Schwarz inequality applied many times. Although it is easy to say when the Schwarz inequality is strict, the multiple application of the Schwarz inequality makes it difficult to say when inequality (2.1) is strict. Analysis of specific problems can shed some light on whether inequality $(2.1)$ is strict.

The proof of Theorem 1 depends on writing the fraction $\mathrm{k} / \mathrm{m}$ as a repeating binary "decimal". The reason for a binary representation is that the Schwarz inequality is based on squares. Lemmas A and B are used to prove identical inequalities for parts (i) and (ii) of Theorem 1 respectively. Lemma $C$ extends the result of Lemmas A and B. Finally the result of Lemma $\mathrm{C}$ is enough to prove Theorem 1 with relative ease. 
Lemma A. Assume the hypotheses of Theorem 1(i) and that $(u, u)=1$. If $k$ and $m$ are integers which satisfy $0 \leq k<m \leq n$ and $m$ is even then

$$
\left|\left(A^{k} u, u\right)\right| \leq\left|\left(A^{r} u, u\right)\right|^{1 / 2}\left|\left(A^{m} u, u\right)\right|^{q / 2}
$$

holds. Here $q$ and $r$ are the unique integers with $0 \leq r<m$ which satisfy $2 k=m q+r$.

Proof of Lemma A. The division algorithm guarantees the existence of $r$ and $q$ as defined. Since $2 k<2 m, q$ must be either zero or one.

Case (i) $(q=0)$. If $q=0$ then $2 k=r<m$. In this case we have

$$
\begin{aligned}
\left|\left(A^{k} u, u\right)\right| & \leq\left|\left(A^{k} u, A^{k} u\right)\right|^{1 / 2}=\left|\left(A^{2 k} u, u\right)\right|^{1 / 2}\left|\left(A^{m} u, u\right)\right|^{0} \\
& =\left|\left(A^{r} u, u\right)\right|^{1 / 2}\left|\left(A^{m} u, u\right)\right|^{q / 2} .
\end{aligned}
$$

Of course the inequality is the Schwarz inequality and the equalities are assumed by hypothesis.

Case (ii) $(q=1)$. If $q=1$ then $r=2 k-m$. In this case we have

$$
\begin{aligned}
\left|\left(A^{k} u, u\right)\right| & =\left|\left(A^{k-(m / 2)} u, A^{m / 2} u\right)\right| \\
& \leq\left|\left(A^{k-(m / 2)} u, A^{k-(m / 2)} u\right)\right|^{1 / 2}\left|\left(A^{m / 2} u, A^{m / 2} u\right)\right|^{1 / 2} \\
& =\left|\left(A^{2 k-m} u, u\right)\right|^{1 / 2}\left|\left(A^{m} u, u\right)\right|^{1 / 2}=\left|\left(A^{r} u, u\right)\right|^{1 / 2}\left|\left(A^{m} u, u\right)\right|^{q / 2} .
\end{aligned}
$$

In Lemma B we prove the same thing but with the hypotheses of part (ii) of Theorem 1.

Lemma B. Assume the hypotheses of Theorem 1(ii) and $(u, u)=1$. If $k$ and $m$ are integers which satisfy $0 \leq k<m \leq n$ and $m$ is odd then inequality (2.2) holds. Here $q$ and $r$ are the unique integers with $0 \leq r<m$ which satisfy $2 k=m q+r$.

Proof of Lemma B. Again the division algorithm guarantees the existence of $r$ and $q$ as defined. Again there are two cases determined by the value of $q$.

Case (i) $(q=0)$. If $q=0$ we have $2 k=r<m$. In this case we have

$$
\left|\left(A^{k} u, u\right)\right| \leq\left|\left(A^{k} u, A^{k} u\right)\right|^{1 / 2}=\left|\left(A^{2 k} u, u\right)\right|^{1 / 2}=\left|\left(A^{r} u, u\right)\right|^{1 / 2}\left|\left(A^{m} u, u\right)\right|^{q / 2} \text {. }
$$

Case (ii) $(q=1)$. If $q=1$ we have $r=2 k-m$. In this case we have

$$
\begin{aligned}
\left|\left(A^{k} u, u\right)\right|= & \left|\sum_{j=1}^{M}\left(B_{j} A^{k-(m+1) / 2} u, B_{j} A^{(m-1) / 2} u\right)\right| \\
\leq & \left(\sum_{j=1}^{M}\left(B_{j} A^{k-(m+1) / 2} u, B_{j} A^{k-(m+1) / 2} u\right)\right)^{1 / 2} \\
& \times\left(\sum_{j=1}^{M}\left(B_{j} A^{(m-1) / 2} u, B_{j} A^{(m-1) / 2} u\right)\right)^{1 / 2} \\
= & \left|\left(A^{2 k-m} u, u\right)\right|^{1 / 2}\left|\left(A^{m} u, u\right)\right|^{1 / 2}=\left|\left(A^{r} u, u\right)\right|^{1 / 2}\left|\left(A^{m} u, u\right)\right|^{q / 2} .
\end{aligned}
$$


We will use the common result of Lemmas A and B, namely, inequality (2.2) to prove Lemma C.

Lemma C. Assume the hypotheses of Theorem 1 and that $(u, u)=1$. Let a be any positive integer. For typographical convenience, let $\tau=2^{-\alpha}$. If $k$ and $m$ are integers which satisfy $0 \leq k<m \leq n$ then the inequality

$$
\left|\left(A^{k} u, u\right)\right| \leq\left|\left(A^{r} u, u\right)\right|^{\tau}\left|\left(A^{m} u, u\right)\right|^{q \tau}
$$

holds where $q$ are $r$ are the unique integers with $0 \leq r<m$ which satisfy $2^{\alpha} k=m q+r$.

Remark. Notice that when $\alpha=1$, inequality (2.3) reduces to inequality (2.2).

Proof of Lemma C. Again $r$ and $q$ exist and are unique by the division algorithm. As remarked above, for $\alpha=1$, inequality (2.3) is established by Lemmas A and B. We proceed by induction on $\alpha$. Suppose that inequality (2.3) holds for some positive $\alpha$ with $\tau=2^{-\alpha}$. By hypothesis we have $2^{\alpha} k=m q+r$ so equivalently $k=r \tau+q m \tau$. The division algorithm again guarantees the existence and uniqueness of integers $r^{\prime}$ and $q^{\prime}$ with $0 \leq r^{\prime}<m$ which satisfy $2 r=r^{\prime}+q^{\prime} m$. Since $r<m$ we may use whichever of Lemma A or B is applicable according to the value of $m$ to obtain the inequality

$$
\left|\left(A^{r} u, u\right)\right| \leq\left|\left(A^{r^{\prime}} u, u\right)\right|^{1 / 2}\left|\left(A^{m} u, u\right)\right|^{q^{\prime} / 2}
$$

which is no more that inequality (2.2) with $k, r$, and $q$ replaced with $r, r^{\prime}$, and $q^{\prime}$ respectively. Subsitution of (2.4) into the supposed valid (2.3) yields the inequality

$$
\left|\left(A^{k} u, u\right)\right| \leq\left[\left|\left(A^{r^{\prime}} u, u\right)\right|^{1 / 2}\left|\left(A^{m} u, u\right)\right|^{q^{\prime} / 2}\right]^{\tau}\left|\left(A^{m} u, u\right)\right|^{q \tau}
$$

or

$$
\left|\left(A^{k} u, u\right)\right| \leq\left|\left(A^{r^{\prime}} u, u\right)\right|^{\tau / 2}\left|\left(A^{m} u, u\right)\right|^{\left(2 q+q^{\prime}\right) \tau / 2} .
$$

This will complete the proof if we can show that (i) $0 \leq r^{\prime}<m$, and (ii) $2^{(\alpha+1)} k=m\left(2 q+q^{\prime}\right)+r^{\prime}$. Condition (i) is satisfied by construction. Notice that $2^{\alpha+1}=2 / \tau$ and that $2^{\alpha} k=m q+r$ by hypothesis. Substitution of the latter into the left-hand side of condition (ii) along with equation $r=r^{\prime} / 2+q^{\prime} m / 2$ yields

$$
2(m q+r)=2 m q+2\left(r^{\prime} / 2+q^{\prime} m / 2\right)=2 m q+q^{\prime} m+r^{\prime}=m\left(2 q+q^{\prime}\right)+r^{\prime} .
$$

Thus inequality (2.3) holds for all positive integers $\alpha$.

At this point we have developed enough to complete the proof of Theorem 1 with relative ease. Since $\mathrm{k} / \mathrm{m}$ is a rational number, we can write it as a repeating binary "decimal". Such an expansion consists of an initial "block" followed by infinitely many (repeating) "blocks" which are identical. We will write $\mathrm{k} / \mathrm{m}$ in a fashion to suggest that fact. After writing $\mathrm{k} / \mathrm{m}$ in this manner we apply Lemma $C$ to the "blocks" to obtain the result. 
Proof of Theorem 1. Suppose that $(u, u)=1$. First observe that if $k=0$ or $k=m$ the result is trivial so we may suppose that $0<k<m$. As $k / m$ is rational, we may write it in the form

$$
\frac{k}{m}=s 2^{-s^{\prime}}+t 2^{-s^{\prime}} \sum_{i=1}^{\infty} 2^{-t^{\prime} i} \text {. }
$$

The numbers $s, s^{\prime}, t$, and $t^{\prime}$ are nonnegative integers. Furthermore $s^{\prime}$ may be chosen to be the "length" of the nonrepeating "block" and $t^{\prime}$ may be chosen to be the "length" of the repeating "block". The numbers $s^{\prime}$ and $t^{\prime}$ are not unique but both may be chosen strictly less than $m$ and at least 1 . The numbers $s$ and $t$ should be thought of as the "value" of each "block", nonrepeating and repeating respectively. We may, and in fact will, assume that $t$ is chosen so that the term $t \sum_{i=1}^{\infty} 2^{-t^{\prime} i}<1$. This merely guarantees that there is no "carry" from the term with the infinite sum to the term $s 2^{-s^{\prime}}$. If $t$ were chosen so that $t \sum_{i=1}^{\infty} 2^{-t^{\prime} i} \geq 1$ then we could simply choose $s$ larger by the amount that $t \sum_{i=1}^{\infty} 2^{-t^{\prime} i}$ exceeds one.

For typographical convenience we again define $\sigma=2^{-s^{\prime}}$ and $\tau=\sum_{i=1}^{\infty} 2^{-t^{\prime} i}$. With these definitions we can rewrite (2.5) as $k=m s \sigma+m \sigma t \tau$. Equivalently and more conveniently we have

$$
k \sigma^{-1}=k 2^{s^{\prime}}=m s+m t \tau .
$$

First observe that the product $m t \tau$ is an integer since $k 2^{s^{\prime}}$ and $m s$ are both integers. Secondly the prodcut $m t \tau<m$ since it was assumed above that $t$ was chosen so that $t \tau<1$. In particular equation (2.6) satisfies the requirements of Lemma $\mathrm{C}$. Therefore we have the inequality

$$
\left|\left(A^{k} u, u\right)\right| \leq\left|\left(A^{m t \tau} u, u\right)\right|^{\sigma}\left|\left(A^{m} u, u\right)\right|^{s \sigma}
$$

which follows from inequality (2.3) of Lemma C.

The next thing to do is to obtain an inequality for the factor $\left|\left(A^{m t \tau} u, u\right)\right|$. We rewrite the quantity $m t \tau$ as follows:

$$
m t \tau=m t \sum_{i=1}^{\infty} 2^{-t^{\prime} i}=m t 2^{-t^{\prime}} \sum_{i=1}^{\infty} 2^{-t^{\prime} i}+m t 2^{-t^{\prime}} .
$$

For our purposes it is best to rewrite $(2.8)$ as

$$
m t \tau 2^{t^{\prime}}=m t \tau+m t=m(t)+m t \tau
$$

We have already established that $m t \tau$ is an integer less than $m$ and therefore Lemma $\mathrm{C}$ is applicable with (2.9). Hence the inequality

$$
\left|\left(A^{m t \tau} u, u\right)\right| \leq\left|\left(A^{m t \tau} u, u\right)\right|^{\delta}\left|\left(A^{m} u, u\right)\right|^{t \delta}
$$

holds, where we have set $\delta=2^{-t^{\prime}}$. If we solve inequality $(2.10)$ for $\left|\left(A^{m t \tau} u, u\right)\right|$, we obtain the inequality

$$
\left|\left(A^{m t \tau} u, u\right)\right| \leq\left|\left(A^{m} u, u\right)\right|^{t \delta /(1-\delta)}
$$


Placing inequality (2.11) into inequality (2.7) yields the inequality

$$
\left|\left(A^{k} u, u\right)\right| \leq\left|\left(A^{m} u, u\right)\right|^{\sigma t \delta /(1-\delta)}\left|\left(A^{m} u, u\right)\right|^{s \sigma}
$$

or equivalently

$$
\left|\left(A^{k} u, u\right)\right| \leq\left|\left(A^{m} u, u\right)\right|^{s \sigma+\sigma t \delta /(1-\delta)} .
$$

The proof is complete if we can show that the quantity $s \sigma+\sigma t \delta /(1-\delta)$ is the same as $\mathrm{k} / \mathrm{m}$. But this is not difficult as equation (2.6) implies that $k / m=s \sigma+t \tau \sigma$. Also the definitions of $\tau$ and $\delta$ are related by $\tau=\sum_{i=1}^{\infty} \delta^{i}=$ $\delta /(1-\delta)$. Hence equality (2.6) and the definition of $\tau$ and $\delta$ imply that $k / m=s \sigma+\sigma t \delta /(1-\delta)$.

In the event that $0<(u, u) \neq 1$ we can always normalize $u$ and apply the above proof to $v=u /\|u\|$. Then the result is that

$$
\left|\left(A^{k} u, u\right)\right|\|u\|^{-2} \leq\left|\left(A^{m} u, u\right)\right|^{k / m}\|u\|^{-2 k / m}
$$

or

$$
\left|\left(A^{k} u, u\right)\right| \leq\left|\left(A^{m} u, u\right)\right|^{k / m}\|u\|^{2(m-k) / m}=\left.\left|\left(A^{m} u, u\right)\right|^{k / m}(u, u)\right|^{(m-k) / m} .
$$

Finally, if $u=0$, the result is trivial.

Examples. We will see some more interesting implications of Theorem 1 later but for now we can look at some simple applications. In the following assume that the inner product space consist of two orthonormal vectors $u$ and $v$. In this case $(u+v, u+v)=2$.

(i) Equality need not hold in (2.1) even if $A$ is selfadjoint. For example suppose that $A u=u$ and $A v=2 v$; then

$$
(A(u+v), u+v)=3<5^{1 / 2} \cdot 2^{1 / 2}=\left(A^{2}(u+v), u+v\right)^{1 / 2}(u+v, u+v)^{1 / 2} .
$$

(ii) Inequality (2.1) may be reversed if $A$ is not selfadjoint. For example suppose that $A u=u$ and $A v=u+v$; then

$$
(A(u+v), u+v)=1+1+1=3>\left(A^{2}(u+v), u+v\right)^{1 / 2}(u+v, u+v)^{1 / 2} \approx 2.828 .
$$

(iii) Even if $A$ is selfadjoint, part (ii) of Theorem 1 may fail. Consider the operator $A$ given by $A u=-2 u$ and $A v=v$; then

$$
\left.\left(A^{2}(u+v), u+v\right)=5>\mid A^{3}(u+v), u+v\right)\left.\right|^{2 / 3}(u+v, u+v)^{1 / 2} \approx 4.6 .
$$

It is interesting to notice that none of the linearity assumed explicitly in Theorem 1 is necessary. One can prove the following completely abstract theorem for which the results are identical except for notation.

Theorem 2. Let $\mathbf{R}_{+}=\{x \in \mathbf{R}: x \geq 0\}$. Let $X$ be any set and $D$ a subset of $X$. Let $A$ and $\psi$ be functions: $A: D \rightarrow X$ and $\psi: X \times X \rightarrow \mathbf{R}_{+}$. Suppose that $\psi$ satisfies

$$
\psi(x, y)^{2} \leq \psi(x, x) \psi(y, y) \text { for all } x, y \in D
$$


Suppose that $n$ is a positive integer and $u$ any fixed element of the domain of $A^{n}$. Finally suppose that for all integers $p$ and $r$ which satisfy $0 \leq r \leq p \leq n$ the equation

$$
\psi\left(A^{p} \cdot u, u\right)=\psi\left(A^{p-r} u, A^{r} u\right)
$$

holds. Then

(i) for each integer $k$ and even integer $m$ which satisfy $0 \leq k \leq m \leq n$, the inequality

$$
\psi\left(A^{k} u, u\right) \leq \psi\left(A^{m} u, u\right)^{k / m} \psi(u, u)^{(m-k) / m}
$$

holds.

(ii) Inequality (2.15) holds for each odd integer $m$ and integer $k$ which satisfy $0 \leq k \leq m \leq n$ if in addition to the above hypotheses the following conditions are satisfied: There is a function $\eta: X \times X \rightarrow \mathbf{R}_{+}$so that for each pair $x, y \in D$ the inequality

$$
\eta(x, y)^{2} \leq \eta(x, x) \eta(y, y)
$$

is satisfied and if for any integers $p$ and $r$ which satisfy $1 \leq r \leq p \leq n$ the equality

$$
\psi\left(A^{p} u, u\right)=\eta\left(A^{p-r} u, A^{r-1} u\right)
$$

holds.

Discussion of Theorem 2. A proof of Theorem 2 can be found in [5] for the case $\psi(u, u)=1$. However the proof follows the one given for Theorem 1 using the lemmas. To prove Theorem 2 , inequality $(2.2)$ becomes

$$
\psi\left(A^{k} u, u\right) \leq\left.\psi\left(A^{r} u, u\right)^{1 / 2}\left[\frac{\psi\left(A^{m} u, u\right)}{\psi(u, u)}\right]^{q / 2} \psi(u, u)\right|^{1 / 2},
$$

and inequality (2.3) becomes

$$
\psi\left(A^{k} u, u\right) \leq\left.\psi\left(A^{r} u, u\right)^{\tau}\left[\frac{\psi\left(A^{m} u, u\right)}{\psi(u, u)}\right]^{q \tau} \psi(u, u)\right|^{1-\tau} .
$$

The remainder of the proof follows easily following the method of proof for Theorem 1 using the above changes. It is interesting to note that we are able to establish the same result for which we assumed homogeneity to obtain the result of Theorem 1 for nonunit vectors $u$ without any explicit assumption of homogeneity or in fact any structure on the set $X$ at all.

Remark. (i) Notice that in Theorems 1 and 2 the functions $B_{j}$ and $\eta$ respectively may depend on the choice of the vector $u$. In our applications, however, the functions $B_{j}$ will be the same regardless of the choice of $u$.

(ii) While Theorem 2 is somewhat abstract, it does point out what is really required. First some sort of "Schwarz inequality" as in (2.13) and (2.16) is required. Secondly, a condition of "symmetry" in $A$ is required by equations (2.14) and (2.17). 


\section{INEQUALITIES FOR EIGENVALUES OF RELATED OPERATORS}

In This section we will give some general eigenvalue comparison theorems for selfadjoint problems related through a single operator. These results use the result of Theorem 1. First we will recall some basic facts.

Definition. Let $H$ be a complex Hilbert space. Let $D$ be a submanifold of $H$. A symmetric operator $T: D \rightarrow H$ is called semibounded below if there is a real constant $c$ so that $(T u, u) \geq c(u, u)$ for all $u \in D$.

Theorem 3. Let $H$ be a complex Hilbert space and $D \subseteq H$ be a dense linear submanifold. Suppose that the linear operator $T: D \rightarrow H$ is selfadjoint and semibounded below with discrete spectrum of finite multiplicity: $\lambda_{1} \leq \lambda_{2} \leq \cdots$. Then

$$
\lambda_{1}=\min \{(T u, u):\|u\|=1 \text { and } u \in D\}
$$

and for $n=2,3,4, \ldots$

$$
\lambda_{n}=\min \left\{(T u, u): u \in D,\|u\|=1 \text { and }\left(u, u_{i}\right)=0 \text { for } i=1,2, \ldots, n-1\right\}
$$

where $u_{i}$ is a (nontrivial) eigenvector of $T$ corresponding to $\lambda_{i}$. Moreover, any minimizing vector in (3.1) or (3.2) is an eigenvector of $T$ with corresponding eigenvalue.

Theorem 3 is well known. A proof can be found in Weinstein and Stenger [9] as well as other texts. We next apply Theorem 3 to polynomials in an operator. The combination of Theorems 1 and 4 provide many interesting inequalities for eigenvalues.

Theorem 4. Let $H$ be a complex Hilbert space. Let $p(x)$ be a polynomial in one variable with real coefficients which is nondecreasing for $x \geq 0$. (E.g., the derivative $p^{\prime}(x) \geq 0$ if $x \geq 0$.) Let $l$ be a positive integer. Suppose that $D_{1}$ and $D_{2}$ are dense linear submanifolds of $H$. Finally suppose that $B: D \rightarrow H$ is a linear operator in $H$ and that each of the operators

$$
B^{l} \text { with domain } D_{1}
$$

and

$$
p(B) \text { with domain } D_{2}
$$

is selfadjoint and semibounded below with discrete spectrum of finite multiplicity, $0 \leq \lambda_{1} \leq \lambda_{2} \leq \cdots$ and $\mu_{1} \leq \mu_{2} \leq \cdots$ respectively. then

(i) If $D_{1} \subseteq D_{2}$ and $(p(B) w, w) \leq p\left(\left(B^{l} w, w\right)^{1 / l}\right)$ for each unit vector $w \in D_{1}$

$$
\mu_{i} \leq p\left(\lambda_{i}^{1 / l}\right) \text { for each } i=1,2, \ldots .
$$

Moreover, equality holds in (3.5) for the index $i$ only if there is a vector $w \in D_{1}$ which is simultaneously an eigenvector of problems (3.3) and (3.4) with eigenvalues $\lambda_{i}$ and $\mu_{i}$ respectively. In particular, if $p(B) w=\mu w$ for $w \in D_{1}$ implies that $w=0$ then inequality (3.5) is strict for all $i$. 
(ii) If $D_{2} \subseteq D_{1}$ and $(p(B) w, w) \geq p\left(\left(B^{l} w, w\right)^{1 / l}\right)$ for each unit vector $w \in$ $D_{2}$ then

$$
\mu_{i} \geq p\left(\lambda_{i}^{1 / l}\right) \text { for each } i=1,2, \ldots .
$$

Moreover, equality holds in (3.6) for the index $i$ only if there is some $w \in D_{2}$ which is simultaneously an eigenvector of problems (3.3) and (3.4) with eigenvalues $\lambda_{i}$ and $\mu_{i}$ respectively. In particular, if $B^{l} w=\lambda w$ for $w \in D_{2}$ implies that $w=0$ then inequality (3.6) is strict for each $i$.

Proof of Theorem 4. Parts (i) and (ii) of Theorem 4 are essentially the same except that the inclusions and inequalities are reversed. The proof of part (ii) is therefore identical to the proof of part (i) except for changing the direction of the inequalities. Thus we will only prove part (i) of Theorem 4.

For $i=1$, let $w_{1}$ be any unit eigenvector of problem (3.3) with eigenvalue $\lambda_{1}$. Then we have

$$
p\left(\lambda_{1}^{1 / l}\right)=p\left(\left(B^{l} w_{1}, w_{1}\right)^{1 / l}\right) \geq\left(p(B) w_{1}, w_{1}\right) \geq \mu_{1} .
$$

The first inequality in (3.7) comes from the hypothesis in part (i) of this theorem. The second inequality in (3.7) comes from Theorem 3 . For $i=2,3$, $\ldots$ we proceed as follows. Let $u_{1}, u_{2}, \ldots, u_{i-1}$ be normalized eigenvectors of problem (3.4) with eigenvalues $\mu_{1}, \mu_{2}, \ldots, \mu_{i-1}$ respectively. Let $w_{1}$, $w_{2}, \ldots, w_{i}$ be normalized eigenvectors of problem (3.3) with eigenvalues $\lambda_{1}$, $\lambda_{2}, \ldots, \lambda_{i}$ respectively. Let $w \sum_{j=1}^{i} \alpha_{j} w_{j}$ where the $\alpha_{j}$ are scalars chosen so that the inner products $\left(w, u_{j}\right)=0$ for $j=1,2, \ldots, i-1$ and so that $\|w\|=1$. This can be done since the problem amounts to finding a nontrivial solution for a homogeneous system of $i-1$ equations in $i$ unknowns. In any case we have

$$
p\left(\lambda_{i}^{1 / l}\right) \geq p\left(\left(B^{l} w, w\right)^{1 / l}\right) \geq(p(B) w, w) \geq \mu_{i} .
$$

The sequence of inequalities (3.8) can be justified as follows. The quantity

$$
\left(B^{l} w, w\right)=\sum_{j=1}^{i}\left|\alpha_{j}\right|^{2} \lambda_{j} \leq \sum_{j=1}^{i}\left|\alpha_{j}\right|^{2} \lambda_{i} \leq \lambda_{i} .
$$

Since the polynomial $p$ is assumed to be nondecreasing for nonnegative argument, we also have $p\left(\left(B^{l} w, w\right)^{1 / l}\right) \leq p\left(\lambda_{i}^{1 / l}\right)$ which is the first inequality of (3.8). The second inequality of (3.8) holds by hypothesis and finally since $w$ is a unit vector orthogonal to each of the first $i-1$ eigenvectors of problem (3.4), Theorem 3 implies the last inequality of (3.8).

If equality holds in (3.5), the last inequality of (3.7) or (3.8), as the case may be, must actually be an equality. According to Theorem 3, the last inequalities of (3.7) and (3.8) are equalities only if the vectors $w_{1}$ and $w$ respectively are eigenvectors of problem (3.4), thus establishing the "moreover" part of this theorem. 
It is most convenient to apply Theorem 4 to elliptic differential operators, $B$, with various boundary conditions and the polynomials taken to have nonnegative coefficients. The inequalities $p\left(\left(B^{l} w, w\right)^{1 / l}\right) \geq(\leq)(p(B) w, w)$ are easily established using Theorem 1. Attempting to list all problems and applications of Theorem 4 would be futile and is in no way limited to the few examples given here. Before we look at some examples we should mention that Theorem 4 is not too interesting if $B$ is a bounded operator as then inequalities (3.5) and (3.6) are equalities by the spectral mapping theorem. Thus, unbounded operators, of which differential operators are the most natural, are the most important and interesting choices for the operator $B$.

Theorem 5. Let $\Omega$ be a bounded domain in $\mathbf{R}^{n}$ with a boundary $\partial \Omega$ and $H=L^{2}(\Omega)$. Suppose that $p(x)=a_{0} \sum_{j=k}^{m} a_{j} x^{j}$ is a polynomial with real coefficients and $a_{j} \geq 0$ for $j=k, k+1, \ldots, m$. Let $A(x) w$ be an $n \times n$ positive definite selfadjoint matrix for each $x \in \Omega$. Consider the operator $B u=$ $-\operatorname{div}(A(x))^{2} \operatorname{grad}(u)$ and let $\partial / \partial n$ be the normal derivative on $\partial \Omega$. Suppose that in $\Omega$, the problems

$$
\begin{gathered}
(B)^{l} u=\lambda u \quad \text { in } \Omega, \quad u=\frac{\partial u}{\partial n}=\cdots=\frac{\partial^{l-1} u}{\partial n^{l-1}}=0 \quad \text { on } \partial \Omega, \\
p(B) v=\mu v \quad \text { in } \Omega, \quad v=\frac{\partial v}{\partial n}=\cdots=\frac{\partial^{m-1}}{\partial n^{m-1}}=0 \quad \text { on } \partial \Omega,
\end{gathered}
$$

and

$$
(B)^{m} w=\nu w \quad \text { in } \Omega, \quad w=\frac{\partial w}{\partial n}=\cdots=\frac{\partial^{m-1} w}{\partial n^{m-1}}=0 \quad \text { on } \partial \Omega
$$

are selfadjoint with discrete spectra of finite multiplicity: $0 \leq \lambda_{1} \leq \lambda_{2} \leq \cdots$; $\mu_{1} \leq \mu_{2} \leq \cdots ; \nu_{1} \leq \nu_{2} \leq \cdots$. If $l \leq k$ then we have

$$
p\left(\lambda_{i}^{1 / l}\right) \leq \mu_{i} \leq p\left(\nu_{i}^{1 / m}\right) \text { for all } i
$$

In fact, if $l<m$, the strict inequality $p\left(\lambda_{i}^{1 / l}\right)<\mu_{i}$ holds for each $i$.

Proof of Theorem 5. To see why inequality (3.12) holds, we notice that in Theorem 4 we can choose $D_{1}$ to be all vectors in $H$ which satisfy the boundary conditions of (3.9). (More precisely, $D_{1}$ is the domain of the closure of the operator $(B)^{l}$ with domain given by the boundary conditions.) We let $D_{2}$ be the domain for problem (3.10) constructed as $D_{1}$ was above and $D_{3}$ the domain of problem (3.11) constructed as above. Thus we have $D_{1} \supseteq D_{2} \supseteq D_{3}$. Notice that $p(x)$ is nondecreasing for $x \geq 0$ since its coefficients except for the constant term are nonnegative.

We utilize Theorem 1 to establish inequalities $(p(B) w, w) \leq p\left(\left(B^{m} w, w\right)^{1 / m}\right)$ and $(p(B) v, v) \geq p\left(\left(B^{l} v, v\right)^{1 / l}\right)$ for each unit vector $w \in D_{3}$ and unit vector $v \in D_{2}$. We choose the operator $B$ to be the operator $A$ of Theorem 1. Let $D(x)$ be a positive definite diagonal $n \times n$ matrix for each $x \in \Omega$ and $U(x)$ 
be the (complex) unitary matrix so that $A(x)=U(x)^{*}(D(x))^{2} U(x)$. For each $j=1,2, \ldots, n$, let $B_{j} u=\mathbf{e}_{j} \cdot \operatorname{grad}(D(x) u(x) \operatorname{grad}(u))$ where $\mathbf{e}_{j}$ is the $j$ th standard basis vector in $\mathbf{R}^{n}$. With these choices of $A$ and $B_{j}$ it is easy to show that both parts (i) and (ii) of Theorem 1 are satisfied for $v \in D_{2}$ and $w \in D_{3}$ where the number $n$ of Theorem 1(not to be confused with the dimension of $\mathbf{R}^{n}$ ) is the number $m$ for problems (3.9)-(3.11). That is, for unit vectors $w \in D_{2}$ (or $D_{3}$ ) we have

$$
\left((B)^{j} w, w\right)=\int_{\Omega}(B)^{j} w \cdot w \leq\left((B)^{s} w, w\right)^{j / s}=\left(\int_{\Omega}(B)^{s} w \cdot w\right)^{j / s}
$$

for all integers $j$ and $s$ with $0 \leq j \leq s \leq m$. In particular for unit vectors $w \in D_{3}$ we have

$$
\begin{aligned}
(p(B) w, w) & =a_{0}+\sum_{j=k}^{m} a_{j}\left((B)^{j} w, w\right) \\
& \leq a_{0}+\sum_{j=k}^{m} a_{j}\left(\left((B)^{m} w, w\right)^{1 / m}\right)^{j}=p\left(\left((B)^{m} w, w\right)^{1 / m}\right) .
\end{aligned}
$$

For unit vectors $v \in D_{2}$ we have

$$
\begin{aligned}
p\left(\left((B)^{l} v, v\right)^{1 / l}\right) & =a_{0}+\sum_{j=k}^{m} a_{j}\left((B)^{l} v, v\right)^{j / l} \\
& \leq a_{0}+\sum_{j=k}^{m} a_{j}\left((B)^{j} v, v\right)=(p(B) v, v) .
\end{aligned}
$$

Therefore all of the hypotheses of Theorem 4 are satisfied and thus inequality (3.12) holds. The strict inequality $p\left(\lambda_{i}^{1 / l}\right)<\mu_{i}$ holds since it is well known that if $l<m$ and $(B)^{l} u=\lambda u$ in $\Omega$ and $u$ and its first $m-1$ derivatives are zero on the boundary of $\Omega$ then $u$ must be zero. Hence the "moreover" part of Theorem 4 is applicable.

We easily have

Corollary. For the collection of problems

$$
(-\Delta)^{k} u=\lambda^{(k)} u \quad \text { in } \Omega ; \quad u=\frac{\partial u}{\partial n}=\cdots=\frac{\partial^{k-1} u}{\partial n^{k-1}}=0 \quad \text { on } \partial \Omega
$$

with eigenvalues $\lambda_{1}^{(k)} \leq \lambda_{2}^{(k)} \leq \cdots$, if $\Omega \subseteq \mathbf{R}^{n}$ is such that problems (3.14) are selfadjoint with spectra of finite multiplicity then we have

$$
\lambda_{i}^{(k)}<\left(\lambda_{i}^{(j)}\right)^{k / j} \text { for all } i \text { if } k<j .
$$

Examples. (i) We can consider problems similar to Theorem 5 with negative coefficients also, but one cannot give a completely general result. Consider the 
problems

$$
\begin{gathered}
-\Delta u=\lambda u \quad \text { in } \Omega ; \quad u=0 \quad \text { on } \partial \Omega ; \\
\Delta^{2} v+2 a \Delta v=\mu v \quad \text { in } \Omega ; \quad v=\partial v / \partial n=0 \quad \text { on } \partial \Omega ;
\end{gathered}
$$

and

$$
\Delta^{2} w=\Gamma w \quad \text { in } \Omega ; \quad w=\partial w / \partial n=0 \quad \text { in } \partial \Omega .
$$

Suppose that problems (3.16), (3.17), and (3.18) are selfadjoint with discrete spectra of finite multiplicity where $\lambda_{1} \geq a>0$. For problems $(3.16)-(3.18)$ the inequalities

$$
\mu_{i}>\lambda_{i}^{2}-2 a \lambda_{i}
$$

and

$$
\mu_{i}+2 a\left(\mu_{i}+a^{2}\right)^{1 / 2}+2 a^{2} \geq \Gamma_{i}
$$

hold for each index $i$.

To see why (3.19) and (3.20) hold we rewrite (3.16)-(3.18) as follows:

$$
\begin{aligned}
(-\Delta-a) u=(\lambda-a) u \quad \text { in } \Omega ; & u=0 \quad \text { on } \partial \Omega ; \\
(-\Delta-a)^{2} v=\left(\mu+a^{2}\right) v \quad \text { in } \Omega ; & v=\partial v / \partial n=0 \quad \text { on } \partial \Omega ;
\end{aligned}
$$

and

$$
\begin{aligned}
& (-\Delta-a)^{2} w+2 a(-\Delta-a) w=\left(\Gamma-a^{2}\right) w \quad \text { in } \Omega \\
& w=\partial w / \partial n=0 \quad \text { on } \partial \Omega .
\end{aligned}
$$

The operator $(-\Delta-a)$ satisfies part (i) of Theorem 1 for vectors satisfying the boundary conditions of problem (3.22). Evidently the eigenvalues of (3.21)(3.23) are $\lambda_{i}-a, \mu_{i}+a^{2}$, and $\Gamma_{i}-a^{2}$ respectively. Application of the method of Theorem 5 yields

$$
\mu_{i}+a^{2}>\left(\lambda_{i}-a\right)^{2}=\lambda_{i}^{2}-2 a \lambda_{i}+a^{2} .
$$

Thus $\mu_{i}>\lambda_{i}^{2}-2 a \lambda_{i}$. Also we have

$$
\left(\mu_{i}+a^{2}\right)+2 a\left(\mu_{i}+a^{2}\right)^{1 / 2} \geq \Gamma_{i}-a^{2} .
$$

This yields the inequality

$$
\mu_{i}+2 a\left(\mu_{i}+a^{2}\right)^{1 / 2}+2 a^{2} \geq \Gamma_{i} .
$$

An upper bound for the eigenvalues of (3.17) may also be obtained through the following more general example.

(ii) Suppose that $p$ and $q$ are polynomials with real nonnegative coefficients with the degree of $p$ greater than the degree of $q$. Suppose that $\operatorname{deg}(p)=m$. Consider the two problems

$$
(-\Delta)^{m} v=\Gamma v \quad \text { in } \Omega ; \quad v=\frac{\partial v}{\partial n}=\cdots=\frac{\partial^{m-1} v}{\partial n^{m-1}}=0 \quad \text { on } \partial \Omega
$$




$$
\begin{aligned}
& (p(-\Delta)-q(-\Delta)) w=\mu w \quad \text { in } \Omega ; \\
& w=\frac{\partial w}{\partial n}=\cdots=\frac{\partial^{m-1}}{\partial n^{m-1}}=0 \quad \text { on } \partial \Omega .
\end{aligned}
$$

The eigenvalues of (3.24) and (3.25) satisfy

$$
\mu_{i} \leq p\left(\left(\Gamma_{i}\right)^{1 / m}\right)
$$

for each index $i$. In particular for (3.17) and (3.18) the eigenvalues satisfy $\mu_{i} \leq \Gamma_{i}$.

To see the result (3.26), we modify Theorem 4 slightly. For $\mu_{i}$ we are interested in minimizing the inner product $((p(-\Delta)-q(-\Delta)) v, v)$ where $v$ satisfies the boundary conditions of (3.25). Then we use part (i) of Theorem 4 with the modified inequality

$$
((p(-\Delta)-q(-\Delta)) v, v) \leq(p(-\Delta) v, v) \leq p\left(\left((-\Delta)^{m} v, v\right)^{1 / m}\right) .
$$

The remainder follows in the manner of the proof of Theorem 4.

As a final example for this section we can consider certain "weighted" eigenvalue problems. One of the most notable is the buckling problem $\Delta^{2} w+\nu \Delta w=$ 0 in $\Omega$ with $w=\partial w / \partial n=0$ on $\partial \Omega$.

(iii) Consider the two problems

$$
(-\Delta)^{m} u=\lambda(-\Delta)^{l} u \quad \text { in } \Omega ; \quad u=\frac{\partial u}{\partial n}=\cdots=\frac{\partial^{m-1} v}{\partial n^{m-1}}=0 \quad \text { on } \partial \Omega
$$

and

$$
(-\Delta)^{m} v=\mu v \quad \text { in } \Omega ; \quad v=\frac{\partial v}{\partial n}=\cdots=\frac{\partial^{m-1} v}{\partial n^{m-1}}=0 \quad \text { on } \partial \Omega
$$

where $l<m$. Then the eigenvalues of (3.27) and (3.28) satisfy

$$
\lambda_{i} \geq\left(\mu_{i}\right)^{(m-l) / m} .
$$

To show why (3.29) holds, we note that in Theorem 3 we consider "unweighted" eigenvalue problems of the type of problem (3.28) where we could write the right-hand side as $\mu \operatorname{Identity}(v)$. For the "weighted" problem an operator other than the identity is chosen as in (3.27). In the event that the weighted problem (3.27) is considered in which a complete set of eigenvectors exists, the $i$ th eigenvalue is characterized by minimizing the ratio

$$
\frac{\left((-\Delta)^{m} v, v\right)}{\left((-\Delta)^{l} v, v\right)}
$$

where $v$ satisfies

$$
\left(v,(-\Delta)^{l} v_{j}\right)=0 \text { for } j=1,2, \ldots, i-1
$$

in which $(-\Delta)^{m} v_{j}=\lambda_{j}(-\Delta)^{l} v_{j}$ and $v_{j} \neq 0$. 
As we have seen in previous examples, in general we will only need to show that the quantity in (3.30) is at least

$$
\left((-\Delta)^{m} v, v\right)^{(m-l) / m}
$$

To do this we choose any unit vector $v$ which satisfies the boundary conditions of (3.27) and apply Theorem 1. But

$$
\frac{\left((-\Delta)^{m} v, v\right)}{\left((-\Delta)^{l} v, v\right)} \geq \frac{\left((-\Delta)^{m} v, v\right)}{\left((-\Delta)^{m} v, v\right)^{l / m}}=\left((-\Delta)^{m} v, v\right)^{(m-l) / m} .
$$

Using the fact that the quantity in (3.30) is at least as large as the quantity in (3.32) and an argument similar to the proof of Theorem 4 except that the "weighted" orthogonality condition (3.31) is used in place of the usual orthogonality condition when constructing the test vector $w$ for $i \geq 2$, we obtain the desired result (3.29).

In particular for the three problems

$$
\begin{array}{lll}
\Delta^{2} w & =\Gamma w \quad \text { in } \Omega ; & w=\partial w / \partial n=0 \quad \text { on } \partial \Omega ; \\
-\Delta u=\lambda u \quad \text { in } \Omega ; & u=0 \quad \text { on } \partial \Omega ;
\end{array}
$$

and

$$
\Delta^{2} v=-\nu \Delta v \quad \text { in } \Omega ; \quad v=\partial v / \partial n=0 \quad \text { on } \partial \Omega,
$$

the eigenvalues satisfy

$$
\lambda_{i}^{2}<\Gamma_{i} \leq \nu_{i}^{2}
$$

Notice that Theorem 5 and the results of (3.27)-(3.29) generally will provide a sequence of inequalities similar to (3.33).

We can use inequality (3.29) to extend and improve a result established by Levine and Protter [6] for problem (3.27) with $m=2$ and $l=1$. In [6], lower bounds for eigenvalues were considered. Among these results are:

(i) For

$$
(-\Delta)^{m} u=\lambda^{(m)} u \quad \text { in } \Omega \subseteq \mathbf{R}^{n}, \quad u=\frac{\partial u}{\partial n}=\cdots=\frac{\partial^{m-1} u}{\partial n^{m-1}}=0 \quad \text { on } \partial \Omega,
$$

the eigenvalues satisfy

$$
\lambda_{i}^{(m)} \geq \frac{n}{n+2 m}\left[\frac{(2 \pi)^{n} i}{B_{n} V}\right]^{2 m / n} .
$$

Here $V$ is the volume of $\Omega$ in $\mathbf{R}^{n}$ and $B_{n}$ is the volume of the unit ball in $\mathbf{R}^{n}$; and

(ii) For

$$
(-\Delta)^{2} v=\mu(-\Delta) v \quad \text { in } \Omega \subseteq \mathbf{R}^{n} ; \quad v=\partial v / \partial n=0 \quad \text { on } \partial \Omega,
$$

the eigenvalues satisfy

$$
\mu_{i} \geq \frac{n}{n+2}\left[\frac{(2 \pi)^{n} i}{n B_{n} V}\right]^{2 / n}
$$


Inequality (3.33) implies that

$$
\begin{aligned}
\mu_{i} & \geq\left(\lambda_{i}^{(2)}\right)^{1 / 2} \geq\left[\frac{n}{n+4}\left[\frac{(2 \pi)^{n} i}{B_{n} V}\right]^{4 / n}\right]^{1 / 2} \\
& =\left[\frac{n}{n+4}\right]^{1 / 2}\left[\frac{(2 \pi)^{n} i}{B_{n} V}\right]^{2 / n} .
\end{aligned}
$$

Inequality (3.38) is stronger than inequality (3.37) since

$$
\frac{n}{n+4}>\left[\frac{n}{n+2}\right]^{2} \cdot \frac{1}{n^{4 / n}}
$$

for all positive integers $n$.

Using the result (3.29) and the more general version of (3.36), (3.27), we can extend inequality (3.38). For the eigenvalue problem

$$
(-\Delta)^{m} v=\mu^{(m, l)}(-\Delta)^{l} v \quad \text { in } \Omega \subset \mathbf{R}^{n} ; \quad v=\frac{\partial v}{\partial n}=\cdots=\frac{\partial^{m-1} v}{\partial n^{m-1}}=0 \quad \text { on } \partial \Omega,
$$

the eigenvalues $\mu^{(m, l)}$ satisfy

$$
\mu_{i}^{(m, l)} \geq\left[\frac{n}{n+2 m}\right]^{(m-l) / m}\left[\frac{(2 \pi)^{n} i}{B_{n} V}\right]^{2(m-l) / n} .
$$

Inequality (3.40) follows as a straightforward combination of inequalities (3.29) and (3.35).

At this point it should be clear what some of the applications of Theorems 1 and 4 are. In general one cannot easily provide an exhaustive list of all of the applications, particularly in a short paper. In order to keep things simple we have only considered one type of boundary condition and in most of the examples the basic operator has been the Laplace operator. In closing this section we make the following remarks.

1. Most of the preceding results are applicable to the same problems but which have the more general elliptic operator of Theorem 5 in place of the Laplace operator.

2. There are many other types of boundary conditions in various combinations to consider. Basically, if the combination of the operator and boundary conditions satisfy both parts of Theorem 1, most of the results of the preceding examples will carry over.

3. No mention here has been made to systems of equations. There is no reason to exclude consideration of systems except for the sake of simplicity. Again, the most significant conditions to satisfy for a system are those which Theorem 1 requires. 


\section{OTHER INEQUALITIES FOR SELFADJOINT OPERATORS}

The inequalities of $\S 3$ are for operators which are related through a single operator. We can also obtain inequalities for eigenvalues of operators which need not be related through a single operator. The principal theorem of this section is a generalization of the method of Rayleigh-Ritz which is effectively expressed by Theorem 3 . We will begin with a few definitions.

Definition. Let $C^{n}$ be $n$-dimensional complex space with its usual inner product $\prec \cdot, \cdot \succ$. Let $M$ be any collection of $n \times n$ complex matrices. Let $A$ and $B$ be matrices that lie in $M$. We say that $A \geq B$ if the inner product $\prec(A-B) u, u \succ \geq 0$, for all $u \in C^{n}$. If $f$ is a function $f: M \rightarrow \mathbf{R}$ for which $f(A) \geq f(B)$ whenever $A, B \in M$ and $A \geq B$, then we say that $f$ is nondecreasing on $M$.

Definition. Again let $M$ be any collection of $n \times n$ complex matrices. Suppose that $f: M \rightarrow \mathbf{R}$ is a function. If for all $A, B \in M$ which are unitarily equivalent by a unitary $n \times n$ matrix, which need not lie in $M, f(A)=f(B)$ then we say that $f$ is invariant under unitary equivalence.

Theorem 6. Let $H$ be a complex Hilbert space with inner product $(\cdot, \cdot)$ and $D$ a dense linear submanifold. Suppose that $T: D \rightarrow H$ is selfadjoint and semibounded below with discrete spectrum of finite multiplicity $\lambda_{1} \leq \lambda_{2} \leq \cdots$. Let $n$ be a positive integer. Define the set of $n \times n$ matrices

$$
E=\left\{\left(\left(T p_{i}, p_{j}\right)\right):\left\{p_{i}\right\}_{i=1}^{n} \text { is an orthonormal set in } D\right\} .
$$

Let $\wedge$ be the $n \times n$ diagonal matrix with the first $n$ eigenvalues $\lambda_{1}, \lambda_{2}, \ldots, \lambda_{n}$ on its diagonal in order from the upper left to the lower right. Suppose that $f: E \rightarrow \mathbf{R}$ is a function which is invariant under unitary equivalence and nondecreasing on $E$. Then

$$
f(\wedge)=\min \{f(A): A \in E\} .
$$

Proof of Theorem 6. First notice that the eigenvectors of $T$ exist in $D$ and are orthogonal. So suppose we consider the orthonormal set $\left\{v_{i}\right\}_{i=1}^{n}$ in $D$ so that $T v_{i}=\lambda_{i} v_{i}$. Then the matrix $\left(\left(T v_{i} v_{j}\right)\right)=\wedge \in E$. Thus $\wedge \in E$ and so the value $f(\wedge)$ is obtained for some $A \in E$, namely $A=\wedge$. Therefore it suffices to show that

$$
f(\wedge) \leq f(A)
$$

for all $A \in E$.

With the objective expressed by inequality (4.3) in mind, choose any matrix $A \in E$ and a corresponding orthonormal set $\left\{p_{i}\right\}_{i=1}^{n}$. Let $N$ be the span of the vectors $\left\{p_{i}\right\}_{i=1}^{n}$ and the linear operator $P$ be the selfadjoint projection of $H$ onto $N$. Define the operator $T_{0}: N \rightarrow N$ by restricting the operator PTP: $H \rightarrow N$ to the subspace $N$. Notice that, in fact $T_{0}$ is selfadjoint and for a fixed basis for $N$ can be represented by an $n \times n$ matrix which lies in 
$E$. In particular for the basis $\left\{p_{i}\right\}_{i=1}^{n}$ above, the matrix for $T_{0}$ is exactly the matrix $A$. In any case, $T_{0}$ has an orthonormal set of eigenvectors $\left\{w_{i}\right\}_{i=1}^{n}$ and corresponding eigenvalues $\mu_{1} \leq \mu_{2} \leq \cdots \leq \mu_{n}$.

Notice that $w_{1} \in N \subseteq D$ with unit norm. Thus $\lambda_{1} \leq\left(T w_{1}, w_{1}\right)=\left(P T P w_{1}\right.$, $\left.w_{1}\right)=\left(T_{0} w_{1}, w_{1}\right)=\mu_{1}$.

For $j=2,3, \ldots, n$, define the vector $w=\sum_{i=1}^{j} \alpha_{i} w_{i}$ in which the $\alpha_{i}$ are chosen so that $\left(w, v_{i}\right)=0$ for $i=1,2, \ldots, j-1$ and so that $\|w\|=1$. Again this is possible, as in the proof of Theorem 4, since finding the $\alpha_{i}$ amount to finding a nontrivial solution for $j-1$ linear equations in $j$ unknowns. With $w$ as above we have

$$
\lambda_{j} \leq(T w, w)=(P T P w, w)=\left(T_{0} w, w\right)=\sum_{i=1}^{j}\left|\alpha_{i}\right|^{2} \mu_{i} \leq u_{j}
$$

since $w$ is orthogonal to the first $j-1$ eigenvectors of $T$.

To complete the proof, observe that the diagonal matrix $M$ with the eigenvalues $\mu_{1}, \mu_{2}, \ldots, \mu_{n}$ on its diagonal in order from the upper left to the lower right is greater than or equal to the matrix $\wedge$, as shown above, and unitarily equivalent to the matrix $A$. Thus $f(\wedge) \leq f(M)=f(A)$ which establishes inequality (4.3) and completes the proof.

Examples. (i) Suppose that the operator $T$ in Theorem 6 is positive with $0<\lambda_{1} \leq \lambda_{2} \leq \cdots$. It is well known that the characteristic polynomial for an $n \times n$ matrix is invariant under unitary equivalence as well as under any similarity transformation. In particular, the absolute value of each coefficient function of the characteristic polynomial, which is a symmetric function in $n$ variables, is invariant under unitary equivalence and nondecreasing. Therefore, for a positive operator $T, f$ any coefficient function for the characteristic polynomial of an $n \times n$ matrix and the $\lambda_{i}$ and $\mu_{i}$ as in the proof of Theorem 6 we have $f\left(\lambda_{1}, \lambda_{2}, \ldots, \lambda_{n}\right) \leq f\left(\mu_{1}, \mu_{2}, \ldots, \mu_{n}\right)$. So $f$ is nondecreasing and invariant under unitary equivalence and is applicable to Theorem 6 .

(ii) Consider an operator $T$ as in example (i) above which for the moment is not necessarily positive and any orthonormal set $\left\{p_{1}, p_{2}\right\} \subseteq D$. Suppose that $\lambda_{1} \geq \alpha$. Then

$$
\lambda_{2} \leq\left(T p_{1}, p_{1}\right)+\left(T p_{2}, p_{2}\right)-\alpha .
$$

Here we use Theorem 6 with $f$ the trace of $2 \times 2$ matrices which is one of the coefficients of the characteristic polynomial and is applicable in Theorem 6 according to example (i). Thus Theorem 6 implies that $\alpha+\lambda_{2} \leq \lambda_{1}+\lambda_{2} \leq$ $\left(T p_{1}, p_{1}\right)+\left(T p_{2}, p_{2}\right)$ or $\lambda_{2} \leq\left(T p_{1}, p_{1}\right)+\left(T p_{2}, p_{2}\right)-\alpha$. Thus a lower bound for $\lambda_{1}$ yields an upper bound for $\lambda_{2}$. Conversely, a similar proof shows that if $\alpha$ is a lower bound for $\lambda_{2}$, we may replace $\lambda_{2}$ with $\lambda_{1}$ in inequality (4.4) yielding an upper bound for $\lambda_{1}$. This, of course, is a hard way to solve an easy problem as upper bounds for $\lambda_{1}$ are easily obtained anyway. 
(iii) Consider an operator $T$ as in examples (i) and (ii) above which is now assumed positive. Suppose that we have $0<\alpha \leq \lambda_{1} \leq \lambda_{2}$ and $\left\{p_{1}, p_{2}\right\} \subseteq D$ is any orthonormal set. Then

$$
\lambda_{2} \leq \min \left\{\left(T p_{1}, p_{1}\right)+\left(T p_{2}, p_{2}\right)-\alpha,\left(T p_{1}, p_{1}\right) \cdot\left(T p_{2}, p_{2}\right) \alpha^{-1}\right\}
$$

We established half of inequality (4.5) in example (ii) above so we need only show that $\lambda_{2} \leq\left(T p_{1}, p_{1}\right) \cdot\left(T p_{2}, p_{2}\right) \cdot \alpha^{-1}$. We have $\alpha \lambda_{2} \leq \lambda_{1} \lambda_{2} \leq\left(T p_{1}, p_{1}\right)$ - $\left(T p_{2}, p_{2}\right)$ by Theorem 6 and example (i) above. Since $\alpha$ is positive this becomes $\lambda_{2} \leq\left(T p_{1}, p_{1}\right)\left(T p_{2}, p_{2}\right) \cdot \alpha^{-1}$ as required.

(iv) Assume $T$ is as in Theorem 6. The method of Rayleigh-Ritz essentially states that

$$
\lambda_{1}=\min \{(T p, p):\|p\|=1 \text { and } p \in D\}
$$

and

(4.7) $\lambda_{n}=\min \{(T p, p): p \in D,\|p\|=1$ and $p$ is orthogonal to each of the first $n-1$ eigenvectors of $T\}$.

This method follows from Theorem 6 using $f(A)=$ trace of $A$ as discussed in example (i). To see this just take $p_{i}=u_{i}$ for $i=1,2, \ldots, n-1$ where $u_{i}$ is a unit eigenvector of $T$ corresponding to the eigenvalue $\lambda_{i}$ and $p_{n}$ any unit vector orthogonal to the $u_{i}$ for $i=1,2, \ldots, n-1$. Then Theorem 6 implies that

$$
\operatorname{trace}(\wedge) \leq\left(T p_{1}, p_{1}\right)+\cdots+\left(T p_{n}, p_{n}\right)=\lambda_{1}+\lambda_{2}+\cdots+\lambda_{n-1}+\left(T p_{n}, p_{n}\right)
$$

Hence we have

$$
\lambda_{1}+\lambda_{2}+\cdots+\lambda_{n} \leq \lambda_{1}+\lambda_{2}+\cdots+\left(T p_{n}, p_{n}\right) \text { or } \lambda_{n} \leq\left(T p_{n}, p_{n}\right) .
$$

Thus the right-hand side of (4.7) is at least as large as the left-hand side. Equality is attained in (4.7) with the choice $p_{n}=u_{n}$ and $u_{n}$ is a unit eigenvector of $T$ corresponding to $\lambda_{n}$. Equation (4.6) is established similarly.

We also have the following theorem which is a generalization of an earlier result of Payne [7] which follows from Theorem 6 and has several interesting implications.

Theorem 7. Let $H$ be a complex Hilbert space. Let $D_{A+B}, D_{A}$, and $D_{B}$ be dense linear submanifolds of $H$ for which $D_{A+B} \subseteq D_{A} \cap D_{B}$. Suppose that each of the three operators

$$
\begin{gathered}
A: D_{A} \rightarrow H, \\
B: D_{B} \rightarrow H, \\
A+B\left(\text { restricted to } D_{A+B}\right): D_{A+B} \rightarrow H
\end{gathered}
$$


is a linear selfadjoint operator in $H$ semibounded below with discrete spectrum of finite multiplicity. Denote the spectrum of each of (4.8), (4.9), and (4.10) by $\lambda_{1} \leq \lambda_{2} \leq \cdots, \mu_{1} \leq \mu_{2} \leq \cdots$, and $\omega_{1} \leq \omega_{2} \leq \cdots$ respectively. Then

$$
\sum_{i=1}^{n} \omega_{i} \geq \sum_{i=1}^{n} \lambda_{i}+\sum_{i=1}^{n} \mu_{i}
$$

for each positive integer $n$.

Proof of Theorem 7. Choose any positive integer $n$. In the following, the sets $\left\{p_{i}\right\}_{i=1}^{n}$ are orthonormal sets of vectors in the submanifold indicated. We have

$$
\begin{aligned}
\sum_{i=1}^{n} \omega_{i} & =\min \left\{\sum_{i=1}^{n}\left((A+B) p_{i}, p_{i}\right): p_{i} \in D_{A+B}\right\} \\
& \geq \min \left\{\sum_{i=1}^{n}\left(A p_{i}, p_{i}\right): p_{i} \in D_{A+B}\right\}+\min \left\{\sum_{i=1}^{n}\left(B p_{i}, p_{i}\right): p_{i} \in D_{A+B}\right\} \\
& \geq \min \left\{\sum_{i=1}^{n}\left(A p_{i}, p_{i}\right): p_{i} \in D_{A}\right\}+\min \left\{\sum_{i=1}^{n}\left(B p_{i}, p_{i}\right): p_{i} \in D_{B}\right\} \\
& =\sum_{i=1}^{n} \lambda_{i}+\sum_{i=1}^{n} \mu_{i} .
\end{aligned}
$$

The equalities are from Theorem 6 taking $f(A)=\operatorname{trace}(A)$. The first inequality follows from the fact that the minimum of a sum is at least the sum of the minima taken separately. The second inequality follows from the inclusion $D_{A+B} \subseteq D_{A} \cap D_{B}$.

At this point we will look at several applications of Theorem 7 .

Examples. (i) In [7], Payne considered the problems

$$
\Delta u+\lambda u=0 \quad \text { in } \Omega ; \quad u=0 \quad \text { on } \partial \Omega ;
$$

$$
\Delta^{2} \varphi-\mu \varphi=0 \quad \text { in } \Omega ; \quad \varphi=\partial \varphi / \partial n=0 \quad \text { on } \partial \Omega ;
$$

and

$$
\begin{aligned}
& \Delta^{2} \psi-\alpha \Delta \psi-\Gamma \psi=0 \quad \text { in } \Omega \\
& \psi=\partial \psi / \partial n=0 \text { on } \partial \Omega \text { where } \alpha \text { is a constant } \geq 0 .
\end{aligned}
$$

Here $\Omega$ is a region in $\mathbf{R}^{m}$ and $\partial \Omega$ is its boundary and $\partial / \partial n$ is the normal derivative on $\partial \Omega$. Payne established the inequality

$$
\sum_{i=1}^{n} \Gamma_{i} \geq \sum_{i=1}^{n}\left(\mu_{i}+\alpha \lambda_{i}\right)
$$

Inequality (4.15) follows easily from Theorem 7 with $A=\Delta^{2}$ and $B=-\alpha \Delta$. As usual, the domains $D_{A}, D_{B}$, and $D_{A+B}$ are determined by the boundary conditions. 
(ii) We can apply Theorem 7 to polynomials as in $\S 3$ which need not have nonnegative coefficients. As a specific example consider the three problems:

$$
-\Delta^{3} u+a \Delta^{2} u+b \Delta u=\lambda u \quad \text { in } \Omega ; \quad u=\frac{\partial u}{\partial n}=\frac{\partial^{2} u}{\partial n^{2}}=0 \quad \text { on } \partial \Omega
$$

$\Delta^{4} v+c \Delta^{3} v+d \Delta^{2} v+e \Delta v=\mu v \quad$ in $\Omega ; \quad v=\frac{\partial v}{\partial n}=\cdots=\frac{\partial^{3} v}{\partial n^{3}}=0 \quad$ on $\partial \Omega$

and

$$
\begin{gathered}
\Delta^{4} w+(c-1) \Delta^{3} w+(d+a) \Delta^{2} w+(b+e) \Delta w=\omega w \quad \text { in } \Omega \\
w=\frac{\partial w}{\partial n}=\cdots=\frac{\partial^{3} w}{\partial n^{3}}=0 \quad \text { in } \partial \Omega .
\end{gathered}
$$

Here $a, b, c, d$ and $e$ are real constants.

If the eigenvalues of (4.16)-(14.18) are discrete as ordered before then they satisfy the inequality

$$
\sum_{i=1}^{n} \omega_{i} \geq \sum_{i=1}^{n}\left(\mu_{i}+\lambda_{i}\right)
$$

As in example (i), the proof of (4.19) follows from Theorem 7.

(iii) The operators used in Theorem 7 need not be related as they are in $\S 3$. Consider the elliptic differential operator $B$ of Theorem 5 in $\S 3$ and the problems

$$
B u=\lambda u \quad \text { in } \Omega ; \quad u=0 \text { on } \partial \Omega
$$

and

$$
\Delta^{2} v=\mu v \quad \text { in } \Omega ; \quad v=\partial v / \partial n=0 \quad \text { on } \partial \Omega
$$

If the eigenvalues in (4.20)-(4.22) are discrete then

$$
\sum_{i=1}^{n} \Gamma_{i} \geq \sum_{i=1}^{n}\left(\mu_{i}+\lambda_{i}\right)
$$

for all positive $n$. Again, this inequality follows from Theorem 7 as the results of examples (i) and (ii) do.

(iv) Some of the things that can happen with Theorem 7 can be seen with the following linear operators represented by their matrices with respect to an orthonormal basis. Let

$$
A=\left[\begin{array}{ll}
1 & 0 \\
0 & 2
\end{array}\right] \text { and } B=\left[\begin{array}{ll}
2 & 1 \\
1 & 2
\end{array}\right]
$$

Then $A+B=\left[\begin{array}{ll}3 & 1 \\ 1 & 4\end{array}\right]$. Label the eigenvalues of $A$ by $\lambda_{1}$ and $\lambda_{2} ;$ of $B$ by $\mu_{1}$ and $\mu_{2}$; and of $A+B$ by $\Gamma_{1}$ and $\Gamma_{2}$. 
It is easy to see that $\lambda_{1}=1 \quad \lambda_{2}=2, \mu_{1}=1, \mu_{2}=3, \Gamma_{2}=\frac{1}{2}(7+\sqrt{5}) \approx 4.62$. It is a standard fact that $\operatorname{trace}(A+B)=\operatorname{trace}(A)+\operatorname{trace}(B)$. We also have $\Gamma_{1} \approx 2.38>\lambda_{1}+\mu_{1}=2$. The fact that $\Gamma_{1} \geq \lambda_{1}+\mu_{1}$ is implied by inequality (4.11) with $n=1$. This example demonstrates that strict inequality in (4.11) is possible. We also note that $4.62 \approx \Gamma_{2}<\mu_{2}+\lambda_{2}=5$. Thus we cannot conclude in general that $\Gamma_{i} \geq \mu_{i}+\lambda_{i}$ for each index $i$.

As noted in $\S 3$, it is likewise impossible here to give an exhaustive list of all applications of Theorems 6 and 7. These examples are merely a few of their applications. We note that if we consider related operators as we did in our applications of Theorem 4 in $\S 3$ we get inequalities for eigenvalues for each index taken separately. If we fail, as in examples (iii) and (iv) above, to require that the operators be related, we do it at the expense of obtaining inequalities like those of $\S 3$ and must settle for the slightly less pleasing inequality (4.11). However we conclude this paper with an example and a proposed problem.

Example. As we have seen earlier, for the problems

$$
\begin{gathered}
-\Delta u=\lambda u \quad \text { in } \Omega ; \quad u=0 \quad \text { on } \partial \Omega ; \\
\Delta^{2} v=\Gamma v \quad \text { in } \Omega ; \quad v=\partial v / \partial n=0 \quad \text { on } \partial \Omega ;
\end{gathered}
$$

and

$$
\Delta^{2} w-\alpha \Delta w=\mu w \quad \text { in } \Omega ; \quad w=\partial w / \partial n=0 \quad \text { on } \partial \Omega,
$$

the equalities

$$
\lambda_{i}^{2}+\alpha \lambda_{i}<\mu_{i} \leq \Gamma_{i}+\alpha\left(\Gamma_{i}\right)^{1 / 2}
$$

and

$$
\Gamma_{1}+\alpha \lambda_{1} \leq \mu_{1}
$$

hold. The problem here is to establish whether the inequality

$$
\Gamma_{i}+\alpha \lambda_{i} \leq \mu_{i}
$$

holds for each index $i$ in addition to the case $i=1$, which is stronger than the left-hand inequality of (4.26).

The second problem related to the above is to establish additional hypotheses on Theorem 7 so that the inequality

$$
\omega_{i} \geq \lambda_{i}+\mu_{i}
$$

holds for each index $i$ in addition to the index $i=1$. A partial answer is proved in example (iv) above in which the inequality in (4.29) is reversed. In fact, if the trace of $A, B$, and $A+B$ all exist and (4.29) holds, we must have equality in (4.29) for each $i$ since then $\operatorname{trace}(A+B)=\operatorname{trace}(A)+\operatorname{trace}(B)$ and summing (4.29) over all $i$ yields an equality. Hence, any additional hypotheses imposed on Theorem 7 to establish (4.29) must at least imply that the Hilbert space $H$ be infinite-dimensional or that (4.29) is actually an equality for each $i$. 


\section{REFERENCES}

1. R. A. Adams, Sobolev spaces, Academic Press, New York, 1975.

2. Zu-chi Chen, Inequalities for eigenvalues of a class of polyharmonic operators, Applicable Anal. 27 (1988), 289-314.

3. R. Courant and D. Hilbert, Methods of mathematical physics, Vols. I and II, Wiley Interscience, New York, 1953 and 1965.

4. J. B. Diaz, Upper and lower bounds for eigenvalues, Proc. Sympos. Appl. Math., vol. 8, McGraw-Hill, New York, for the Amer. Math. Soc., Providence, R. I., 1958, pp. 53-78.

5. S. M. Hook, Inequalities for eigenvalues of self-adjoint operators, Doctoral Dissertation, Univ. of California, Berkeley, 1986.

6. H. A. Levine and M. H. Protter, Unrestricted lower bounds for eigenvalues for classes of elliptic equations and systems of equations with applications to problems in elasticity, Math. Methods Appl. Sci. 7 (1985), 210-222.

7. L. E. Payne, New isoperimetric inequalities for eigenvalues and other physical quantities, Comm. Pure Appl. Math. 9 (1956), 531-542.

8. A. Weinstein, Etudes des spectres des equations aux derives partielles de la theorie des plaques elastiques, Mem. Sci. Math. 88 (1937).

9. A. Weinstein and W. Stenger, Methods of intermediate problems for eigenvalues, Academic Press, New York, 1972.

Department of Mathematics, University of California, Davis, California 95616

Current address: 869 Rosemont Road, Oakland, California 94610 